\title{
Intra-abdominal fat accumulation predicts the development of the metabolic syndrome in non-diabetic Japanese-Americans
}

\author{
J. Tong • E. J. Boyko $・$ K. M. Utzschneider • \\ M. J. McNeely • T. Hayashi • D. B. Carr • \\ T. M. Wallace $\cdot$ S. Zraika $\cdot$ F. Gerchman • \\ D. L. Leonetti • W. Y. Fujimoto • S. E. Kahn
}

Received: 1 December 2006 / Accepted: 4 February 2007 / Published online: 27 March 2007

(C) Springer-Verlag 2007

\begin{abstract}
Aims/hypothesis Intra-abdominal fat (IAF) is an important risk factor for CHD and type 2 diabetes, and in crosssectional studies is associated with the metabolic syndrome
\end{abstract}

Electronic supplementary material The online version of this article (doi:10.1007/s00125-007-0651-y) contains supplementary material, which is available to authorised users.

J. Tong $(\bowtie) \cdot$ K. M. Utzschneider $\cdot$ T. M. Wallace $\cdot$ S. Zraika $\cdot$

F. Gerchman · S. E. Kahn

Division of Metabolism, Endocrinology and Nutrition,

Department of Medicine, VA Puget Sound Health Care System

(151) and University of Washington,

1660 South Columbian Way,

Seattle, WA 98108, USA

e-mail: tongj@u.washington.edu

\section{E. J. Boyko}

Epidemiologic Research and Information Center,

VA Puget Sound Health Care System,

Seattle, WA, USA

M. J. McNeely · W. Y. Fujimoto

Department of Medicine, University of Washington,

Seattle, WA, USA

\section{T. Hayashi}

Department of Preventive Medicine and Environmental Health,

Osaka City University,

Osaka, Japan

D. B. Carr

Department of Obstetrics and Gynecology,

University of Washington,

Seattle, WA, USA

D. L. Leonetti

Department of Anthropology, University of Washington,

Seattle, WA, USA
(MetS). Our aim was to determine whether IAF accumulation predicts the future development of MetS in nondiabetic Japanese-Americans.

Subjects and methods We conducted a prospective study of 457 Japanese-American men and women (mean \pm SD: age $51.5 \pm 12.0$ years, BMI $23.9 \pm 3.1 \mathrm{~kg} / \mathrm{m}^{2}$ ) without diabetes or MetS at baseline. Of these, 408 completed a 5-year followup and 366 completed a 10-year follow-up. BMI, waist circumference, IAF and subcutaneous fat (SCF) areas by computed tomography, blood pressure, fasting plasma glucose, insulin, triacylglycerol and HDL-cholesterol were measured at baseline and at 5- and 10-year follow-up. MetS was defined using National Cholesterol Education Program Adult Treatment Panel III criteria.

Results Incidence of MetS was $15.3 \%$ at 5 years and $17.8 \%$ at 10 years. A change of $1 \mathrm{SD}$ in IAF area was associated with a 2.1-fold increase in the odds of MetS at 10 years (odds ratio $=2.08,95 \%$ CI 1.41-3.07) after adjusting for age, sex, baseline IAF and the presence of each individual MetS criteria at baseline. This association was independent of changes in fasting insulin and SCF areas.

Conclusions/interpretation We conclude that IAF accumulation over time independently predicts the development of MetS and thus may play an important role in the development of MetS in Japanese-Americans.

Keywords Asians · Epidemiology - Insulin resistance · Metabolic syndrome $\cdot$ Visceral adiposity

$\begin{array}{ll}\text { Abbreviations } \\ \text { HOMA-IR } & \begin{array}{l}\text { homeostasis model assessment of insulin } \\ \text { resistance }\end{array} \\ \text { IAF } & \text { intra-abdominal fat }\end{array}$

Abbreviations

IAF intra-abdominal fat 


\section{MetS metabolic syndrome}

NECP ATP National Cholesterol Education Program

III Adult Treatment Panel III

OR odds ratio

SCF subcutaneous fat

\section{Introduction}

The co-occurrence of metabolic risk factors such as abdominal obesity, hyperglycaemia, hypertension and dyslipidaemia, collectively referred to as the metabolic syndrome (MetS), increases the risk of type 2 diabetes and cardiovascular disease [1]. While the pathophysiology underlying MetS is uncertain, a recent cross-sectional study demonstrated that intra-abdominal fat (IAF) was stronger than insulin sensitivity as a major determinant [2].

In Japanese-Americans, increased IAF at baseline was associated with the future development of IGT, type 2 diabetes, hypertension and CHD [3-5]. We now demonstrate that increasing IAF over time is associated with a higher incidence of MetS independently of subcutaneous fat (SCF) and fasting insulin levels.

\section{Subjects and methods}

Study population Of 509 non-diabetic subjects who participated in the Japanese-American Community Diabetes Study in King County, Washington, between 1983 and 1988 [5], we excluded participants who met the diagnostic criteria for the National Cholesterol Education Program Adult Treatment Panel III (NECP ATP III) criteria for MetS at baseline, leaving 457 second- (Nisei, $n=242$ ) and thirdgeneration (Sansei, $n=215$ ) Japanese-Americans.

Data collection Evaluations were performed at the University of Washington General Clinical Research Center, using a protocol approved by the University of Washington Human Subjects Review Committee.

Metabolic syndrome MetS was defined based on NCEP ATP III criteria [6] and assessed at baseline and at 5 and 10 years.

Since IAF and waist circumference are highly correlated $(r=0.71, p<0.01)$, waist circumference was excluded in multiple logistic regression analyses examining the association between IAF change and the incidence of modified MetS (two or more criteria).

Waist circumference was measured at the level of the umbilicus to the nearest tenth of a centimetre in men and at the natural waistline in women. Plasma triacylglycerol was measured by enzymatic analytical chemistry. HDL-cholesterol was separated by precipitation of the other lipoproteins with dextran- $\mathrm{Mg}^{2+}$ and cholesterol was measured enzymatically. A 75-g oral glucose tolerance test was performed after a 10-h overnight fast. Diabetes was diagnosed if participants were taking oral hypoglycaemic medication or insulin, if the fasting plasma glucose level was $\geq 7.0 \mathrm{mmol} / \mathrm{l}$ and/or if the 2 -h value was $\geq 11.1 \mathrm{mmol} / \mathrm{l}$. Plasma glucose was measured by the glucose oxidase method and plasma insulin by RIA as previously described [4].

Body fat distribution Cross-sectional areas of adipose tissue were quantified using computed tomography with single $10-\mathrm{mm}$ slices of the thorax on inspiration at the level of the
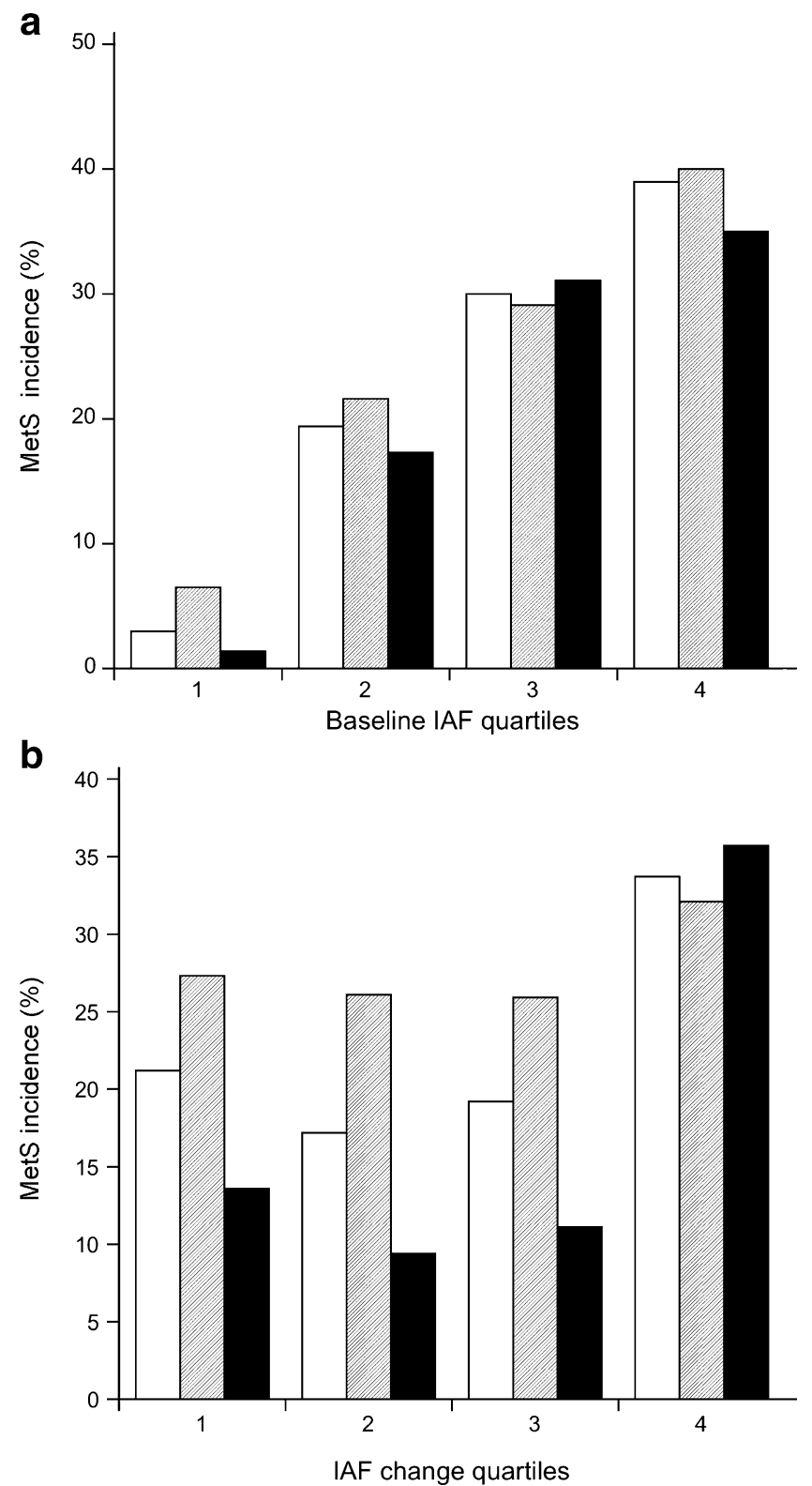

Fig. 1 Incidence of development of MetS after 10 years of follow-up based on baseline IAF quartiles (a) and quartiles of IAF change over time (b). Data are presented for all subjects (open columns), male subjects (grey columns) and female subjects (closed columns) 
nipples, the abdomen at the level of the umbilicus and the mid-thigh [5]. IAF was measured using the transversalis fascia as the outer boundary. Total SCF area was the sum of subcutaneous thoracic and abdominal fat areas and twice the right thigh SCF area.

Statistical analysis Paired and unpaired $t$ tests were used to compare mean values. Multiple logistic regression analysis was used to examine the associations between independent variables such as changes in IAF and SCF areas and fasting insulin over 10 years, and the dependent variable, MetS incidence, dichotomised as yes/no. Results from this analysis are presented as the odds ratio (OR), which was standardised to a 1-SD change for the independent continuous variables. The presence or absence of each individual MetS criterion was adjusted in the regression models to minimise bias related to the greater or lesser likelihood of having a baseline phenotype associated with IAF. We assessed the presence of multi-colinearity by computing the variance inflation factor for the independent variables specified in the regression models. Effect modification was assessed with standard methods involving testing the significance of first-order interaction terms in regression models. All $p$ values reported are two-sided. Values are reported as means \pm SD unless specified otherwise. All statistics were calculated using STATA version 8.0 (STATA, College Station, TX, USA).

\section{Results}

Baseline characteristics After excluding 52 (10.2\%) who met MetS criteria (12.6\% in males and $7.7 \%$ in females), 457 (229 men, mean age 51.4 \pm 11.8 [SD] years and 228 women, mean age $51.6 \pm 12.4$ years) were eligible at baseline. Forty-nine were lost to follow-up after 5-6 years and another 42 between 5 and 10 years. Of the 366 who returned for the 10-year follow-up, only one did not have a complete assessment.

Mean BMI was $23.9 \pm 3.1 \mathrm{~kg} / \mathrm{m}^{2}$. Men had higher BMI and IAF area and women had higher total SCF area and fasting plasma insulin (data not shown). Elevated blood pressure was the most common $(28.9 \%)$ and high waist circumference the least common (3.7\%) criterion at baseline. Participants who completed the 5- or 10-year follow-up had similar body composition and metabolic parameters compared with all participants eligible for the study, as well as those who were lost to follow-up (Electronic Supplementary Material Table 1).

Incidence of MetS Cumulative incidence of MetS was $15.3 \%(61 / 399)$ at 5 years and $17.8 \%(65 / 365)$ at 10 years. MetS status at 10 years was independent of that at 5 years.

The incidence increased as baseline IAF area increased across the IAF quartiles $(p<0.001$ for non-parametric test of trend) (Fig. 1a). During the study, mean IAF area increased
Table 1 Multiple logistic regression analyses using IAF change ( 0 to 10 years) to predict incidence of the modified MetS at 10-year follow-up while adjusting for age, sex and the presence of individual MetS criteria at baseline

ORs for continuous variables reflect a 1 SD magnitude increase

\begin{tabular}{llr}
\hline Independent variables & OR $(95 \% \mathrm{CI})$ & $p$ value \\
\hline Model 1 & & \\
IAF change $\left(\mathrm{cm}^{2}\right)$ & $2.54(1.83-3.52)$ & $<0.001$ \\
Baseline IAF $\left(\mathrm{cm}^{2}\right)$ & $2.29(1.60-3.28)$ & $<0.001$ \\
Age $($ years $)$ & $1.13(0.82-1.55)$ & 0.471 \\
Female sex & $1.33(0.75-2.37)$ & 0.334 \\
High blood pressure & $3.15(1.69-5.85)$ & $<0.001$ \\
High triacylglycerol & $2.86(1.46-5.60)$ & 0.002 \\
Low HDL-cholesterol & $2.93(1.14-7.54)$ & 0.026 \\
High fasting glucose & $3.35(1.68-6.68)$ & 0.001 \\
Model 2 & & $<0.001$ \\
IAF change $\left(\mathrm{cm}^{2}\right)$ & $2.08(1.41-3.07)$ & 0.032 \\
Baseline IAF $\left(\mathrm{cm}^{2}\right)$ & $1.68(1.05-2.69)$ & 0.001 \\
Fasting insulin change $(\mathrm{pmol} / \mathrm{l})$ & $2.27(1.42-3.63)$ & 0.006 \\
Baseline fasting insulin $(\mathrm{pmol} / \mathrm{l})$ & $1.97(1.22-3.19)$ & 0.706 \\
SCF change $\left(\mathrm{cm}^{2}\right)$ & $1.07(0.74-1.55)$ & 0.837 \\
Baseline total SCF $\left(\mathrm{cm}^{2}\right)$ & $1.05(0.67-1.65)$ & 0.113 \\
Age $($ years $)$ & $1.36(0.93-1.98)$ & 0.769 \\
Female sex & $1.14(0.48-2.69)$ & 0.001 \\
High blood pressure & $3.24(1.63-6.43)$ & 0.002 \\
High triacylglycerol & $3.24(1.56-6.72)$ & 0.058 \\
Low HDL-cholesterol & $2.71(0.97-7.59)$ & 0.003 \\
High fasting glucose & $2.98(1.44-6.14)$ & \\
\hline
\end{tabular}


from $89.2 \pm 49.1 \mathrm{~cm}^{2}$ at baseline to $112.9 \pm 57.7 \mathrm{~cm}^{2}$ at 10 years in men; and from $62.3 \pm 38.8 \mathrm{~cm}^{2}$ at baseline to $75.6 \pm$ $39.7 \mathrm{~cm}^{2}$ at 10 years in women.

Association of change in IAF over time and development of MetS The incidence of MetS at 10-year follow-up increased across the quartiles of IAF change over time $(p=0.04)$ (Fig. 1b). Subjects in the first quartile of IAF change had the highest mean baseline IAF compared with those in the second, third and fourth quartiles (100.4 vs 69.2, 65.1 and $71.6 \mathrm{~cm}^{2}$, respectively).

The association between change in IAF area over time and the incidence of modified MetS ( $\geq 2$ ATP III criteria excluding waist circumference) was estimated by multiple logistic regression analysis (Table 1). For every 1-SD $\left(35.8 \mathrm{~cm}^{2}\right)$ or $1-\mathrm{cm}^{2}$ change in IAF area from baseline, there was increased risk of developing MetS at 10 years $(\mathrm{OR}=2.54, p<0.001$ and $\mathrm{OR}=1.02, p<0.001$, respectively), (Table 1, Model 1) after adjusting for baseline IAF area, age, sex and the presence/absence of each individual MetS criterion. Results were similar with the unmodified MetS criteria, which included waist circumference $(\mathrm{OR}=2.04$, $p<0.001)$. These associations were independent of changes in fasting insulin and SCF area (Table 1, Model 2). Baseline fasting insulin level also independently predicted the incidence of MetS at 10 years. Substituting fasting insulin levels with the HOMA-IR (homeostasis model assessment of insulin resistance) index, or total SCF area with abdominal SCF or BMI did not change the association between IAF and incident MetS (results not shown). None of the variance inflation factors for the models shown in Table 1 exceeded 10, thereby making multi-colinearity unlikely [7]. There was no evidence of effect modification between IAF and either sex or generation (Nisei vs Sansei) (results not shown).

\section{Discussion}

In this prospective study of Japanese-Americans who did not have diabetes or MetS at baseline, we found that both the change in IAF area over time and baseline IAF area predicted the future development of MetS. This association appeared to be independent of changes in fasting insulin levels and SCF area.

Prospective studies suggested that high WHR predicts development of MetS [8] but WHR includes both IAF and abdominal wall SCF, two metabolically distinct fat depots. Our findings extend previous prospective and crosssectional studies showing increased visceral fat to be associated with a higher incidence and prevalence of MetS $[2,8]$ by examining the differential effects of IAF vs SCF.
When the concept of MetS was first described, insulin resistance was initially thought to be the primary aetiological process [9]. We observed that both baseline fasting insulin and change in fasting insulin were strong predictors of MetS development at 10 years (Table 1, Model 2). Hence insulin resistance as measured by either fasting insulin or HOMA-IR and IAF accumulation are both good long-term predictors for the development of MetS.

A limitation of our study is the use of fasting insulin as a surrogate marker for insulin sensitivity. A more precise measure of insulin sensitivity [10] may have led to different results. However, Carr et al. used insulin sensitivity from the minimal model and the findings were similar [2]. Since we studied non-obese non-diabetic Japanese-Americans, our findings may not apply to other populations.

In conclusion, both IAF accumulation and increase in fasting insulin are independent predictors of the long-term (10-year) development of MetS in Japanese-Americans and are likely to play important roles in the pathogenesis of MetS.

Acknowledgements We want to thank J. B. Shofer for her assistance with the statistical analysis and review of the manuscript, and R. Hull for her helpful comments. This work was supported by the National Institute of Health grants DK-55460 and DK02860, and the Medical Research Service of the Department of Veterans Affairs. S. E. Kahn was supported in part by an American Diabetes Association Distinguished Clinical Scientist Award.

Duality of interest No author has an interest in or relationship with a company/organization that could benefit financially from the publication of the data in this paper.

\section{References}

1. Stern MP, Haffner SM (1986) Body fat distribution and hyperinsulinemia as risk factors for diabetes and cardiovascular disease. Arteriosclerosis 6:123-130

2. Carr DB, Utzschneider KM, Hull RL et al (2004) Intra-abdominal fat is a major determinant of the National Cholesterol Education Program Adult Treatment Panel III criteria for the metabolic syndrome. Diabetes 53:2087-2094

3. Fujimoto WY, Bergstrom RW, Boyko EJ et al (1999) Visceral adiposity and incident coronary heart disease in JapaneseAmerican men. The 10-year follow-up results of the Seattle Japanese-American Community Diabetes Study. Diabetes Care 22:1808-1812

4. Boyko EJ, Fujimoto WY, Leonetti DL, Newell-Morris L (2000) Visceral adiposity and risk of type 2 diabetes: a prospective study among Japanese Americans. Diabetes Care 23:465-471

5. Hayashi T, Boyko EJ, Leonetti DL et al (2004) Visceral adiposity is an independent predictor of incident hypertension in Japanese Americans. Ann Intern Med 140:992-1000

6. Grundy SM, Cleeman JI, Daniels SR et al (2005) Diagnosis and management of the metabolic syndrome: an American Heart 
Association/National Heart, Lung, and Blood Institute Scientific Statement. Circulation 112:2735-2752

7. Kleinbaum DG, Kupper LL, Muller KE, Nizam A (1998) Applied regression analysis and other multivariable methods. Duxbury, Pacific Grove, CA

8. Han TS, Williams K, Sattar N, Hunt KJ, Lean ME, Haffner SM (2002) Analysis of obesity and hyperinsulinemia in the develop- ment of metabolic syndrome: San Antonio Heart Study. Obes Res 10:923-931

9. Reaven GM (1997) Banting Lecture 1988. Role of insulin resistance in human disease, 1988. Nutrition 13:65, discussion 64, 66

10. Beard JC, Bergman RN, Ward WK, Porte D Jr (1986) The insulin sensitivity index in nondiabetic man. Correlation between clampderived and IVGTT-derived values. Diabetes 35:362-369 